\title{
Ein performatives Konzept im \\ Fremdsprachenunterricht - In 14 Schritten zur eigenen Inszenierung
}

\author{
Am Beispiel einer Kooperation zwischen Staatstheater und \\ universitärem Fremdsprachenunterricht
}

\author{
Bärbel Jogschies, Anke Stöver-Blahak
}

\begin{abstract}
Grundlage für den Beitrag sind Kurse in der Fremdsprache Deutsch am Fachsprachenzentrum der Leibniz Universität Hannover für internationale Studierende aller Fakultäten auf dem Niveau B2 des Gemeinsamen Europäischen Referenzrahmens. Diese Kurse belegen die Teilnehmer*innen freiwillig und studienbegleitend. Sie laufen mit zwei Semesterwochenstunden ca. 13 Wochen. Die Kooperation mit dem Staatstheater besteht in der gemeinsamen Seminarkonzeption, insbesondere der Auswahl von performativen Mitteln und der Planung von Theaterworkshops, Besuchen im Staatstheater hinter den Kulissen und zur Vorstellung. Zum Abschluss der Kurse präsentieren die Studierenden ihre eigene Inszenierung der erarbeiteten Theaterstücke. Die Auswahl der Stücke richtet sich zum einen nach dem Spielplan des Schauspielhauses Hannover. Zum anderen soll es ein originär deutschsprachiges Stück sein - also keine Übersetzung. Gearbeitet wurde bisher mit so unterschiedlichen Aufführungen wie Die Nibelungen (Hebbel), Minna von Barnhelm (Schiller), Am Schwarzen See (Loher), Das Wirtshaus im Spessart (Hauff) und Corpus Delicti (Zeh). Dabei entwickelte sich ein relativ einheitliches Arbeitsmuster (14 Schritte), das den jeweiligen Textgrundlagen, aber auch dem laufenden Spielplan angepasst wurde:
\end{abstract}

\section{Vorbereitung}

Wichtig in der Vorbereitung ist die Fokussierung auf thematische Schwerpunkte, die Auswahl von Schlüsselszenen. Die Erschließung des literarischen Werkes geschieht deduktiv, das heißt vom Detail schrittweise zu einem sich mehr und mehr zusammenfügenden Gesamtbild. Der Ausgangspunkt kann ein thematischer oder ästhetischer sein. Bei der Beschäftigung mit Dea Lohers Am schwarzen See zum Beispiel begannen wir mit der Wahrnehmung der 
unvollständigen Sätze und der fehlenden Interpunktion und schlossen daraus auf eine Stimmung. Bei den Nibelungen war der Beginn das Motiv des Fremdseins in der Figur der Brunhilde. Wichtig ist, dass der Ausgangspunkt genügend Raum für Identifikation oder Distanzwahrnehmung der Lerner*innen bietet. Diese didaktische Arbeit in der Seminarkonzeption gleicht der Lesartfindung zu einem Stück im Theater. Sie ist in aller Regel ein langer Prozess der Auseinandersetzung des Regieteams zu einem Inszenierungsansatz, aus dem sich die Besetzung, die Bühnenbildidee und die Strichfassung erst ergeben. Dieser Ansatz muss immer wieder am Stücktext auf seine Richtigkeit hin überprüft werden und belegbar sein. Schlüsselszenen und Figureninterpretationen ergeben sich aus diesem Ausgangspunkt heraus.

\section{Kennenlernen}

Studierende aus verschiedenen Ländern (Korea, China, Iran, USA, Russland, Frankreich, England etc.), mit unterschiedlichen Muttersprachen und verschiedenen Studienfächern kommen zusammen. Namen müssen gelernt (und gesprochen) werden. Von Beginn an werden die Tische beiseite geräumt. Es gibt einen Stuhlkreis oder aber einen freien Raum. Schon jetzt können Themen der Stücke eingeflochten werden: „Meine Reise nach Deutschland“ Nibelungen, „Bei uns gilt es als gesund, wenn... “ Corpus Delicti, „Mörderspiel“ Wirtshaus im Spessart. Ziel ist es, dass am Ende alle Personen sämtliche Namen sprechen können, dass deutlich wurde, dass verschiedene Kulturen und Herkunftssprachen aufeinandertreffen und der Unterrichtsraum erfahren/erlaufen/entdeckt wurde. Dabei müssen auch das für die meisten fremde Unterrichtskonzept erklärt und Erwartungen geklärt werden. Von vornherein sollen sich die Teilnehmenden an performative Unterrichtsformen gewöhnen, das heißt:

- aktives Handeln statt reiner Rezeption, es wird ausprobiert und nicht ausdiskutiert

- körperliche und emotionale Beteiligung, Warm-ups, körperliche und sprecherische Spiele und Übungen

- Interaktion mit den anderen in Kleingruppen

- ergebnisoffene kreative Darstellungsaufgaben, die der Dozent/die Dozentin nicht reguliert, sondern die in der Gruppe diskutiert werden

- immer wieder Präsentationssituationen mit dem Feedback der Zuschauer*innen und gemeinsamer Reflexion

\section{Thematische Einführung durch Improvisation}

Das Thema des Stückes wird eingeführt. Dabei wird möglichst an die Lebenswelt der Studierenden angeknüpft, z. B. (1) Standbild bauen zum 
Thema Schwarzer See. Nacheinander kommen die Teilnehmer*innen dazu, ergänzen um Adjektive, ergänzen um Geräusche zu ihren Rollen und am Ende erzählen die einzelnen Bildbestandteile, wie sie sich fühlen. (2) Der Dozent/die Dozentin erzählt ein deutsches Märchen. Teilnehmer*innen erzählen zunächst jeweils einen Satz nach und malen dann in Gruppen ein Piktogramm, in dem sie die verschiedenen Stationen des Helden verzeichnen. (3) Wir machen ein gemeinsames Frühstück, jede*r bringt etwas mit, was im Heimatland als „gesund“ gilt. Das Essen wird erklärt, es gibt einen Austausch über gute oder eher ungesunde Gewohnheiten beim Frühstück. Hausaufgabe war, Sprüche zur Gesundheit aus den Heimatländern zu suchen und zu übersetzen. Diese Sprüche und Redensarten werden vorgetragen und diskutiert. Ähnlichkeiten und Überschneidungen sind $\mathrm{zu}$ erwarten. Ziel ist die Aktivierung bzw. Erarbeitung des für das Stück relevanten Wortschatzes, die Herstellung einer Verbindung des Themas zu den Erfahrungen der Teilnehmer*innen (z. B. Essen, aber auch Märchen) und der Aufbau von Vertrauen untereinander durch gemeinsames Handeln. Die Regeln der Improvisation (z. B. Annehmen der Spielvorschläge des Partners/der Partnerin, in der Rolle bleiben, auf Sichtbarkeit für das Publikum achten, deutliches Sprechen, starker körpersprachlicher und emotionaler Ausdruck) finden die Teilnehmenden w ährend d es Tuns o der im Feedback oft selbst. Manchmal sind unterstützenden Hinweise des Dozenten/der Dozentin hilfreich. Grundsätzlich aber gilt: Alles, was die Gruppe selbständig herausfindet, i st $d$ as b essere $u$ nd w eniger $f$ remdbestimmte $W$ issen. $D$ as heißt für den Dozenten/die Dozentin oft Geduld und Zurückhaltung. Je dezenter er/sie Hinweise gibt, desto besser.

\section{Austausch - gemeinsame Improvisation}

Weiterhin wird das Thema des Stückes erarbeitet. Ein Schwerpunkt in dieser Phase ist, dass die Teilnehmer*innen miteinander sprechen und handeln. Dazu können z. B. kleinere Improvisationen erarbeitet werden wie für Corpus Delicti ein Arztbesuch in verschiedenen Ländern (Rollen: Arzt, Patient, Arzthelferin, Begleiter o. ä.), das Nachspielen eines Märchens aus verschiedenen Perspektiven wie für Das Wirtshaus im Spessart oder die Bildung einer Sonderkommission zum Todesfall Am Schwarzen See (Rollen: Kommissar, Zeugen, betroffene Angehörige etc.). Ziel hierbei ist die Festigung und der weitere Ausbau des Wortschatzes. Dies gilt insbesondere in Bezug auf die Interaktion mit den Mitspieler*innen und den Aufbau von Selbstvertrauen der Teilnehmer*innen durch den Gebrauch der Sprache beim Aushandeln, Planen und Durchführen der Improvisationen. In dieser Phase erfahren die Teilnehmenden oftmals die Vielfalt der Umsetzungsmöglichkeiten innerhalb ihrer eigenen Gruppe. Sie lernen, fremde Vorschläge wertzuschätzen, ohne die eigenen negieren zu müssen. Das ist für viele die erste Begegnung mit kollektiver Kreativität und ein wichtiger Gruppenbildungsfaktor: Eine Person allein hätte sich niemals ausdenken können, was in diesem kollektiven Prozess entsteht (Emergenz). Durch diese Erfahrung beginnt eine starke Gruppenbindung und Faszination 
für den Prozess. Man möchte teilhaben, beitragen und nichts verpassen. Das ist wichtig für das künftige Verhalten im Prozess und zur Gruppe.

\section{Texterarbeitung}

Bei der Texterarbeitung steht nicht das vollständige Lesen der Textgrundlage im Vordergrund. Die Erarbeitung ergibt sich aus der Struktur der Vorlage. Es können z. B. Profile zu d en P ersonen d u rch di e A u sgabe vo $\mathrm{n} T$ extschnipseln $\mathrm{z} u$ den einzelnen Charakteren erstellt werden wie in Am Schwarzen See (vier Eltern) und Minna von Barnhelm (Minna). Bei Das Wirtshaus im Spessart wurden die vier verschiedenen enthaltenen Geschichten als Hausaufgabe in Gruppen verteilt. Sie sollten gelesen und theatral nacherzählt werden. Dazu können Techniken wie das Lesen einzelner Wörter im Kreis, das Sprechen von Dialogen im Kreis oder das Chorsprechen angewandt werden. Durch die Begegnung mit dem Text, also der Autorität des Autors, erhöht sich die Bedeutung der eigenen vorherigen thematischen Annäherung. Die zu erfassenden Textpassagen müssen wohldosiert und verständlich sein, also einen Erfolg bescheren („Oh, ich habe Lessing verstanden!"). Gut ist die Arbeit in kleinen Gruppen, die sich selbst organisieren und gegenseitig beim Textverständnis helfen. Ebenso hilfreich ist der Ehrgeiz, dem Rest der Gruppe sein Ergebnis möglichst plastisch, also theatral, als Spielszene zu präsentieren.

\section{Texterarbeitung}

In dieser Sitzung wird die Arbeit der vorherigen fortgeführt. Es wird mit Teilen des Originaltextes gearbeitet, die von den Teilnehmer*innen spielerisch und an Themen bzw. Charakteren orientiert erarbeitet wird. Ziel dieser beiden Sitzungen ist die Einführung des Originaltextes, ohne dass die Studierenden überfordert werden. Durch das schrittweise Erarbeiten gewinnen sie Sicherheit und Selbstvertrauen auch schwierigen und ungewöhnlichen Texten gegenüber (Nibelungen, Minna von Barnhelm, Das Wirtshaus im Spessart). Sie erfahren, dass sie in der Lage sind, solche Texte nicht nur zu rezipieren, sondern sie sogar mit anderen zu gestalten. Es findet ein kollektiver Aneignungsprozess des Werkes statt, bei dem der/die Einzelne nur einen verträglichen Abschnitt zur Texterarbeitung beiträgt, aber teilhat am Diskurs und an den gemeinsamen Versuchen der Verkörperung und Verbildlichung der vorhandenen Vorgänge. Es entsteht ein gemeinsames Wissen, das offen ist für individuelle Meinung und Neigung.

\section{Textarbeit}

Im Fokus steht hier die genaue Textarbeit. Es wird darauf geachtet, dass die Handlung der Vorlagen vollständig verstanden wird. Dazu können, den 
Texten entsprechend, verschiedene Techniken angewandt werden: Die Bewusstmachung der Rahmenhandlung und Schaffung einer Erzählatmosphäre im Wirtshaus im Spessart, die Rekonstruktion der Spielsituation in Am Schwarzen See, das Herausgreifen einer zentralen Szene wie der Gerichtsverhandlung in Corpus Delicti. In allen Fällen wird Theater gespielt. Die Teilnehmer*innen übernehmen Rollen, bewegen sich im Raum, interagieren. Das Ziel ist eine möglichst genaue Kenntnis der Textvorlage vor dem Besuch im Theater. Dies unterscheidet dieses Kurskonzept deutlich von einem Literaturunterricht mit anschließendem Theaterbesuch - der Text wird zwar gelesen, aber kaum intensiv in der Tiefe aufgenommen - sowie von organisierten, aber unvorbereiteten Theaterbesuchen. Die Teilnehmer*innen sollen Textteile so gut kennen, dass sie sie während der Aufführung wiedererkennen und mitsprechen können. Sie sollen sie aber nicht nur kognitiv aufnehmen, sondern mit allen Sinnen erfahren und sozusagen inkorporieren. In dieser Phase werden die Schlüsselszenen des Textes erarbeitet, die zentral für das Verständnis des dramatischen Konfliktes im Stück sind. Wichtig für den Dozenten/die Dozentin ist es, den dramatischen Konflikt möglichst offen zu halten und nicht vorschnell Lösungen anzubieten. So erzeugt er/sie eine kognitive Dissonanz, die Neugier weckt.

\section{Theaterbesuch und Workshop}

Die Workshops orientierten sich an den Vorlagen und an den herausgearbeiteten Themen. Beispielsweise wurden zu Corpus Delicti Kurzvorträge zu den Gesundheitssystemen der verschiedenen Heimatländer gehalten und anschließend ein ideales Gesundheitssystem entworfen. Beim Wirtshaus im Spessart stand das Leben von Wilhelm Hauff im Mittelpunkt, bei Am Schwarzen See das Thema Selbstmord. Der Workshop fand entweder im Unterrichtsraum statt oder im Foyer des Theaters. Alle Theaterbesuche waren verbunden mit einem Besuch hinter der Bühne. Besonders beeindruckend war es für die Teilnehmer*innen, wenn die Vorstellung am Abend schon vorbereitet war und sie sich in dem Bühnenbild bewegen konnten. Das Ziel der Workshops und der Theaterbesuche war eine angstfreie und zwanglose Heranführung der Kursteilnehmer*innen an deutsche Hochkultur. Durch die verschiedenen Phasen der Vorbereitung (Darstellen des Themas in der eigenen Lebenswelt, Texterarbeitung und eigenes erstes Spielen, Aufführung im Theater) sollen sie langsam an verschiedene Formen von Performativität herangeführt werden. Das Erlebnis des Theaters birgt die Begegnung mit einer anderen Gruppe, dem Schauspielensemble, das sich ebenfalls auf performative Weise mit dem Text und dem Autor auseinandergesetzt hat. Die Annahme, dass die höhere Professionalität die Theateranfänger*innen einschüchtert, wird durch den Genuss an der professionellen Schauspielkunst entkräftet. Oftmals sind die Teilnehmer*innen fasziniert und inspiriert. Die für die deutsche Theatertradition typische interpretatorische Freiheit des Theaters gegenüber dem Text führt zur Ermutigung im Hinblick auf die eigene inszenatorische Auseinandersetzung, die in den folgenden Sitzungen stattfinden soll. 


\section{Auswertung des Theaterbesuches - erste Ideen für die eigene Fassung}

Textentstehung aus Improvisation

Durch das intensive Erarbeiten und Sprechen einzelner Textteile und das Sichern des Verständnisses für den Handlungsverlauf konnten die Teilnehmer*innen sowohl der Vorstellung problemlos folgen als auch einzelne Passagen wiedererkennen. Sie waren in der Lage, die Darstellungen der Schauspieler*innen, die Regieanlage und auch das Bühnenbild zu ihren eigenen ersten Spielversuchen mit dem Text, aber auch zu den freien Improvisationen, in Beziehung zu setzen. Dadurch konnten sie Ideen für eine eigene Aufführung gewinnen. Die Ideen wurden kollaborativ aus Standbildern, Nachspielen von Szenen und den Diskussionen über die Theateraufführung entwickelt. Die Geschichten, die auf diese Weise entstanden, waren einerseits stark mit den Ursprungsgeschichten verknüpft (integrierte Originaltextteile). Andererseits entfaltete sich auch ein beeindruckendes kreatives Potential, mit dem völlig eigenständige Abläufe entwickelt wurden. So wurde z. B. in Am Schwarzen See die Perspektive der hinterbliebenen Eltern aufgegeben und die Geschichte nach der Frage „Wie konnte es zu dem Selbstmord der Jugendlichen kommen?“ neu erzählt. Dazu wurden drei mögliche Antworten gegeben, die Handlung wurde also dreimal wieder „zurückgespult“. Die Aufgabe der Dozentin an dieser Stelle ist es, mitzuschreiben, die Notate zu ordnen und mit Teilen der Originaltexte zu einer neuen Textfassung zusammenzufügen. Reicht die Zeit aus, könnte diese Arbeit auch von den Teilnehmer*innen selbst geleistet werden. Das Ziel dieses Arbeitsschrittes ist es, alle Vorbereitungsschritte in eine geplante, bewusst ausgeführte performance münden zu lassen, in der die Teilnehmer*innen die bis dahin gewonnenen Erkenntnisse zum performativen Charakter ihrer Lebenswelten ebenso wie zur Theaterpraxis einfließen lassen können. Aus der Sicht der Theaterpädagogik ist der entstehende Text eine Art Kommentar zur gesehenen Inszenierung. Aus der wahrgenommenen Distanz zwischen Erwartetem und Gesehenem entsteht ein Meinungsbild der Gruppe, die die eigene Interpretation der Theaterinszenierung gegenüberstellt. Die performative Form der Nachbereitung liefert das Stückmaterial. Die Möglichkeiten der szenischen Darstellung der Gruppe sind in aller Regel begrenzter als die des professionellen Theaters. Die Lücken werden durch Erzähltexte gefüllt. Die Form des Erzähltheaters, die Sprechertexte mit szenischen Einschüben kombiniert, bietet sich an. Ebenso geeignet ist eine Szenencollage.

\section{Probe}

Die erste Textfassung der eigenen Aufführung wird zunächst gelesen, besprochen, ergänzt, verändert und die Rollen werden gemeinsam besetzt. Da die Stücke aus Improvisationen heraus entwickelt wurden, bei denen die Teilnehmer*innen ihre Rollen frei wählen konnten, ergeben sich die Zuweisungen 
fast von selbst. Bisher gab es keine Fehlbesetzungen. Im Gegenteil, die Rollen passten zumeist außergewöhnlich gut zu den spielenden Personen. Aufgabe des Dozenten/der Dozentin ist es, während des Schreibprozesses darauf zu achten, dass alle Teilnehmer*innen annähernd gleich lange Sprechzeiten haben. Im Probenprozess ist immer auch ein Teil der Gruppe in der Beobachterrolle, gibt Feedback und macht Vorschläge. Weitere Aufgaben werden vergeben, wie Plakat oder Programmheftgestaltung. Die Feedbackgruppe nimmt die Rolle des zukünftigen Publikums ein und achtet auf die Verständlichkeit der gesamten Darstellung. Erklärt sich die Geschichte auch für Menschen, die den Ausgangstext oder die Inszenierung nicht kennen? Kollegiale Verbesserungsvorschläge richten sich auch auf den gestischen und emotionalen Ausdruck und die Figurenzeichnung. Auch dieser Perspektivwechsel ist Teil des performativen Gesamtkonzeptes.

\section{Probe}

Das Stück wird möglichst mehrmals durchgespielt. Dabei wird parallel das Bühnenbild entwickelt. Vorschläge für Bühnen und Kostümgestaltungen werden in der Gruppe diskutiert und Aufträge vergeben. Die Gruppe ist sich hier bereits der Tatsache bewusst, dass sie ein gemeinsames ästhetisches Erscheinungsbild braucht und dass die verwendeten Gestaltungsmittel nicht illustrierend, sondern zeichenhaft sind. So reicht ein Hut für den Räuber, ein Kopftuch für die Mutter oder ein Plastikumhang für das Glasmännlein. An der Wand hängt ein Steckbrief der Räuber. Ein Tisch mit Biergläsern reicht zur Darstellung einer Wirtshausszene.

\section{Probe}

Das Stück wird mehrmals durchgespielt. Das Ziel der drei Proben ist es, den Teilnehmer*innen Textsicherheit zu geben, damit sie sich zunehmend auf die Bühnenhandlung und das Spiel konzentrieren können. Die Mischung des Textes aus schwierigeren Originalpassagen und zumeist handlungstragenden eigenen, eher umgangssprachlichen Passagen führt dazu, dass auch die Originaltexte flüssig $\mathrm{u} \mathrm{n} \mathrm{d} \mathrm{b}$ e deutungsangemessen $\mathrm{g}$ e sprochen w e rden. Das Thema Lampenfieber, d i e A n gst, v o r a n deren z u s p rechen, w e rden hier bearbeitet. Die Gruppe gibt sich gegenseitig Sicherheit. Die Dozentin ist Souffleuse. J e der D a rsteller/jeder D a rstellerin s o llte w i ssen, w a s i m Falle von Textkonfusion mindestens mitgeteilt werden muss. Hier zeigen sich das gegenseitige Vertrauen der Gruppe und das gemeinsame Wollen.

\section{Aufführung}

Die Aufführung gilt als Semesterleistung und wird mit mehreren Kursen gemeinsam vor einem öffentlichen Publikum durchgeführt. Der Theaterteil hat 
eine Länge von ca. 20 Minuten. Das Ziel ist es, den Studierenden die Möglichkeit $\mathrm{zu}$ eröffnen, die erworbene performative Kompetenz in einem Rahmen einzusetzen, der zwar vorbereitet ist, der aufgrund des unbekannten Publikums jedoch nicht bekannt und daher nur bedingt kalkulierbar ist. Diese Prüfung ist für alle Beteiligten ein Fest: Beweis und Feier des Erworbenen mit großer emotionaler Bedeutung, aber auch mit entsprechendem Leistungsdruck. Wenn möglich, sollte sich die Gruppe vor der Aufführung zu einem gemeinsamen Warm-up mit Lockerungs- und Konzentrationsübungen treffen. Gut ist ein gemeinsames Ermutigungsritual, etwa das deutsche „Toi toi toi!“ oder kleine Premierengeschenke. Die Aufführung vor Publikum wird in aller Regel mit starkem freundschaftlichen Applaus bedacht. Die Spieler*innen erhalten viel Feedback von Studierenden aus anderen Kursen.

\section{Auswertung}

Hier wird die Videoaufzeichnung der Aufführung gemeinsam angesehen und der Kurs insgesamt evaluiert. Es wird zusammengetragen, was die Teilnehmer*innen erfahren und gelernt haben. Wichtige Teilaspekte hierbei sind: Erweiterung des Wortschatzes, Stärkung des Selbstvertrauens beim Sprechen in der fremden Sprache, aber auch beim Sprechen mit und/oder vor anderen Menschen, das Kennenlernen eines deutschen Theaterstücks, der Besuch in einem deutschen Theater, der Austausch der Kulturen mit den anderen Teilnehmer*innen und - dies wird besonders hervorgehoben - die positive Atmosphäre in der Gruppe. Oft berichten die Teilnehmenden stolz vom positiven Feedback durch Freund*innen und Kommiliton*innen. Auch die gegenseitige Wertschätzung und Anerkennung der Leistungsfortschritte, die sie an sich selbst und an anderen wahrgenommen haben, sind eine neue Erfahrung. Da das Kurskonzept den meisten Teilnehmer*innen nicht bekannt ist und diese häufig mit se hrviel En gagement und S paß d a ran teilnehmen, wird leicht übersehen, dass auch sehr viel gelernt wird. Das Ziel ist hier also ein bewusstes Reflektieren der verschiedenen Stationen sowie ein Herausarbeiten des Ertrags - dies stets verbunden mit dem Folgeziel, diesen Ertrag auch für nicht-theatrale Situationen nutzbar zu machen.

F A Z I T - Das Besondere dieses Kurskonzeptes ist, dass es aus verschiedenen Quellen gespeist ist: Es ist Fremdsprachenunterricht, der handlungs- und produktorientiert mit Methoden des Theaters arbeitet und zudem noch einen direkten Zugang zum Theater der Zielsprache ermöglicht. Der vierzehnschrittige Kursverlauf wurde skizziert. Er hat sich in der Praxis bewährt. Die Kooperation zwischen einer universitären Einrichtung und einem Staatstheater wird nicht immer leicht zu realisieren sein, es sind auch andere Formen derZusammenarbeit denkbar. Aber die Weiterführung von performativen Konzepten aus der Unterrichtsumgebung hinaus in Orte, die aus performances, aus Ausführungen 
bestehen, ist lohnenswert. ${ }^{1}$

Stimmen von Studierenden (sprachlich leicht korrigiert): — Zuerst möchte ich das sagen: Es waren fantastische Erfahrungen. Es ist schwierig, dass man Theater vor Leuten in seiner Muttersprache spielt. Aber es ist ungleich schwieriger, dass man ein Theater vor Leuten in einer Fremdsprache spielt. Mit diesem Kurs konnten wir typische deutsche Ausdrücke, Betonung und außerdem Mimik lernen! Und am besten ist: Ich habe mich für das deutsche Theater interessiert. (Jaehyun Lee,Südkorea, E-Mail vom 11.07.2013)

Ich hatte viel Spaß während des Kurses. Das Textbuch war schwierig, aber es gab viele Messages. Ich konnte Deutsch üben. Und meiner Meinung nach hat sich mein Deutsch ein bisschen verbessert. Ich mochte die anderen Studenten, und die Gruppe war sehr schön. (E-Mail vom 16.07.2013) Das war auch eine schöne Erfahrung, dass wir ein Spiel im Theater gesehen haben. Das war das erste Mal, dass ich ein Spiel sehen konnte. Das kann ich nicht vergessen. (Sujin Yoo, Südkorea, E-Mail vom 11.07.2013)

Der Kurs war für mich sehr hilfreich, mich selbstwewusst mit der Sprache zu fühlen. Ich fand die „Aufwärm“-Aktivitäten, die mehr Sprachverwendung erforderten, am besten, z.B. Heimat beschreiben, See-Szene bauen (eine Person ist Baum, die andere Vogel etc., sie waren gut, um Artikel und Adjektive zu üben). Die Führung durch das Staatstheater war wunderbar! (Debra, USA, E-Mail vom 19.07.2013)

In diesem Kurs habe ich die Lautstärke meiner Stimme durch das Theaterspielen geübt.

Die Korrekturen von unserer Dozentin und auch von meinen Kommilitonen führten mich zu einer Ausspracheverbesserung. Toll war es schließlich, dass wir Mimik und Gestik mit einer fremden Sprache kombiniert haben. (Georgia, Griechenland, E-Mail vom 19.07.2013)

\footnotetext{
${ }^{1}$ Videoaufzeichnungen der letzten Aufführungen können unter folgendem Link aufgerufen werden: http://www.fsz.uni-hannover.de/einzelkurse-deutsch.html (Accessed 15 September 2014).
} 